Supporting Information

\title{
Evolutionary gain of alanine mischarging to non-cognate tRNAs with a G4:U69 base pair
}

Litao Sun ${ }^{1}$, Ana Cristina Gomes ${ }^{2}$, Weiwei $\mathrm{He}^{3}$, Huihao Zhou ${ }^{1}$, Xiaoyun Wang ${ }^{2}$, David W. Pan ${ }^{2}$, Paul Schimmel ${ }^{1}$, Tao Pan ${ }^{2 *}$, and Xiang-Lei Yang ${ }^{1,3 *}$

${ }^{1}$ Department of Cell and Molecular Biology, The Scripps Research Institute, La Jolla, CA 92037, USA

${ }^{2}$ Department of Biochemistry and Molecular Biology, University of Chicago, Chicago, IL 60637, USA

${ }^{3}$ Department of Chemical Physiology, The Scripps Research Institute, La Jolla, CA 92037, USA

*Correspondence should be addressed to X.-L.Y. (xlyang@scripps.edu) and T.P. (taopan@uchicago.edu). 


\section{Methods}

\section{Protein expression and purification}

Human full length AlaRS and the truncation AlaRS ${ }^{\mathrm{N} 455}$ (residues 1-455) were cloned into pET-21a and pET-28b vectors, respectively. E.coli AlaRS was cloned into pET-20b vector (Novagen). Constructs were transformed into BL21(DE3) cells. Proteins were sequentially purified by Ni-NTA beads (QIAGEN), Q HP column (GE Healthcare), and HiLoad 16/60 Superdex 200 prep grade column (GE Healthcare). The purified human AlaRS ${ }^{\mathrm{N} 455}$ was collected and concentrated to $20 \mathrm{mg} / \mathrm{ml}$ for crystallization. Human and E.coli AlaRS mutant proteins were constructed by site-directed mutagenesis and purified using the same methods. The final purified proteins were stocked in the $20 \mathrm{mM}$ Tris $\mathrm{pH} 8.0,200 \mathrm{mM} \mathrm{NaCl}$, and $1 \mathrm{mM}$ DTT.

\section{Crystallization and data collection}

High-throughput crystallization screen was performed using Mosquito liquid transfer robot (TTP Labtech). Before setting up crystallization, $1 \mathrm{mM}$ (final concentration) Ala-AMP analog (5'-O-[N-(L-Alanyl)-sulfamoyl] adenosine) AlaSA (Integrated DNA Technologies) was added into human AlaRS ${ }^{\mathrm{N} 455}$ protein solution, and incubated on ice for $1 \mathrm{~h}$. AlaRS ${ }^{\mathrm{N} 455}$ proteins were crystallized with sitting-drop vapor diffusion method. Each drop contained $100 \mathrm{nl}$ of $20 \mathrm{mg} / \mathrm{ml}$ protein and $100 \mathrm{nl}$ reservoir solution, and equilibrated against $70 \mu \mathrm{L}$ reservoir solution. AlaRS ${ }^{\mathrm{N} 455}$ crystals were grown with the reservoir solution (0.1 M Hepes $\mathrm{pH} 7.5,20 \%$ Polyethylene glycol 3350, $0.2 \mathrm{M} \mathrm{MgCl}_{2}$ ) at $16{ }^{\circ} \mathrm{C}$. Crystals were cryoprotected with $15 \%$ glycerol added to the reservoir solution and flash-frozen with liquid nitrogen. Diffraction data were collected at 100 K on beamline 11-1 of Stanford Synchrotron Radiation Lightsource (SSRL), and processed with program HKL2000 ${ }^{1}$.

\section{Structure determination and refinement}

The crystal structure of human $\mathrm{AlaRS}^{\mathrm{N} 455}$ was determined by molecular replacement method using the structure of E. coli AlaRS catalytic domain (PDB 3HXU) as the 
search model and the program MOLREP ${ }^{2}$. The initial model was rebuilt manually in $\operatorname{Coot}^{3}$ according to $2 \mathrm{~F}_{\mathrm{O}}-\mathrm{F}_{\mathrm{C}}$ and $1 \mathrm{~F}_{\mathrm{O}}-\mathrm{F}_{\mathrm{C}}$ maps. Structure refinement was performed with REFMAC $5^{4}$ and PHENIX ${ }^{5}$. The space group is P1 and the final structure was refined to $2.68 \AA$ resolution with $\mathrm{R}_{\text {work }}=21.6 \%$ and $\mathrm{R}_{\text {free }}=25.4 \%$. The structure was validated using PROCHECK ${ }^{6}$. Data collection and structure refinement statistics are summarized in Table 1.

\section{In vitro transcription of tRNAs}

DNA templates containing a T7 promoter and a tRNA gene (tRNA ${ }^{\text {Ala }}$, tRNA ${ }^{\mathrm{Cys}}$, $\mathrm{tRNA}^{\mathrm{Thr}}$, or their mutants) were synthesized by PCR of overlapping oligonucleotides. The transcription reaction was performed in $40 \mathrm{mM}$ Tris- $\mathrm{Cl} \mathrm{pH}$ 8.0, $25 \mathrm{mM} \mathrm{NaCl}, 20$ $\mathrm{mM} \mathrm{MgCl} 2,2 \mu \mathrm{g} / \mathrm{ml}$ pyrophosphatase, $0.1 \mathrm{mg} / \mathrm{ml}$ bovine serum albumin (BSA), 5 mM DTT, $20 \mathrm{mM}$ NTPs with T7 polymerase and the DNA template at $37^{\circ} \mathrm{C}$ for $2 \mathrm{~h}$. The tRNA transcript was purified by phenol-chloroform extraction. The purified tRNA was annealed by heating up to $95{ }^{\circ} \mathrm{C}$ for $3 \mathrm{~min}$ and then slowly cooled down to room temperature with adding $1 \mathrm{mM} \mathrm{MgCl}_{2}$ at $55^{\circ} \mathrm{C}$.

\section{Active site titration assay}

Active site titration assay was performed as described ${ }^{7,8}$ in $100 \mathrm{mM}$ HEPES pH 7.5, $20 \mathrm{mM} \mathrm{KCl}, 10 \mathrm{mM} \mathrm{MgCl} 2,50 \mathrm{mM}$ ATP, $22 \mathrm{nM}\left[\gamma_{-}{ }^{32} \mathrm{P}\right]$-ATP, $20 \mathrm{mM}$ L-Alanine, 2 $\mathrm{mg} / \mathrm{ml}$ pyrophosphatase and $2 \mathrm{mM}$ DTT to determine the concentration of active enzymes.

\section{In vitro aminoacylation assays}

As described previously ${ }^{7}$, the aminoacylation assays were performed at room temperature with $50 \mathrm{mM}$ HEPES pH 7.5, $20 \mathrm{mM} \mathrm{KCl,} 5 \mathrm{mM} \mathrm{MgCl}_{2}, 4 \mathrm{mM}$ ATP, 2 $\mathrm{mM}$ DTT, $4 \mu \mathrm{g} / \mathrm{ml}$ pyrophosphatase, $20 \mu \mathrm{M}$ cold L-alanine, $1.34 \mu \mathrm{M}\left[{ }^{3} \mathrm{H}\right]$-Alanine $(1 \mathrm{mCi} / \mathrm{ml})$ as the assay solution. Various amounts of tRNAs were initially mixed with the assay solution, and the reaction was initiated by adding $200 \mathrm{nM}$ AlaRS protein 
into the mixture. At varying time intervals, $5 \mu \mathrm{l}$ aliquots were applied to M $\mu$ ltiScreen 96-well filter plate (0.45 um pore size Hydrophobic, low protein binding membrane, Merck Millipore Ltd.), which is pre-wetted with quench solution containing 0.5 $\mathrm{mg} / \mathrm{ml}$ DNA and $100 \mathrm{mM}$ EDTA in $300 \mathrm{mM} \mathrm{NaOAc}$ (pH 3.0). After all time points were collected, $100 \mu \mathrm{l} 20 \%$ trichloroacetic acid (TCA) was added to precipitate the nucleic acids. Then the plate was washed four times with $200 \mu$ of $5 \%$ TCA containing $100 \mathrm{mM}$ cold alanine, followed once with 95\% ethanol. On drying after completion of the washing steps, $70 \mu \mathrm{l}$ of $100 \mathrm{mM} \mathrm{NaOH}$ was added to elute the tRNAs, which was then centrifuged into a 96-well flexible PET microplate (PerkinElmer) with $150 \mu \mathrm{l}$ of Supermix scintillation cocktail (PerkinElmer). After mixing, the radioactivity in each well of the plate is counted in the 1450 LSC \& Luminescence Counter (PerkinElmer).

\section{Microarray analysis}

Total RNA was extracted from HeLa cells using TRIzol reagent (Invitrogen), and followed by total tRNA purification by anion-exchange chromatography using a NucleoBond RNA/DNA 400 column (Clontech). Aminoacylation of tRNAs for microarray analysis was performed at room temperature in $20 \mathrm{mM}$ Hepes (pH 7.5), 10 $\mathrm{mM} \mathrm{MgCl}_{2}, 5 \mathrm{mM}$ DTT, $4 \mathrm{mM}$ ATP, $150 \mathrm{mM} \mathrm{NH}_{4} \mathrm{Cl}, 0.1 \mathrm{mM}$ EDTA, $0.37 \mu \mathrm{Ci} / \mu \mathrm{l}$ $\left[{ }^{3} \mathrm{H}\right]-\mathrm{L}-A$ lanine, L-Tyrosine or L-Glycine, $1 \mathrm{mg} / \mathrm{ml}$ total HeLa tRNA and the purified cognate human enzyme (AlaRS, TyrRS, GlyRS or GlyRS- $\Delta$ WHEP). For each $60 \mu 1$ reaction, $30 \mu \mathrm{l}$ of reaction mixture was taken out at $5 \mathrm{~min}$ and $30 \mathrm{~min}$ time points to add ice-cold $1.2 \mu \mathrm{l}$ of $0.5 \mathrm{M}$ EDTA, $30 \mu \mathrm{l}$ of buffer $(0.1 \mathrm{M} \mathrm{NaOAc} / \mathrm{HOAc}, 0.5 \mathrm{M}$ $\mathrm{NaCl}, \mathrm{pH} 4.8)$ and $160 \mu \mathrm{l}$ ethanol to stop the reaction.

The custom tRNA microarray method for tRNA charging has been described previously ${ }^{9,10}$. Each array includes 42 probes for human nuclear encoded tRNA genes and 22 probes for human mitochondrial encoded tRNA genes. The oligonucleotide probes were sequences complementary to the full-length tRNA sequences. Nonhuman tRNA probes were used as specificity controls for hybridization. Eight replicates of each probe were printed on each array. The oligonucleotide probes were printed 
manually to generate array spots necessary for radioactive detection. For array hybridization, radio-labeled tRNA samples were dissolved in $2 x S S C, p H 4.8$. This was then applied to the hybridization chamber of the GeneTAC Hyb4 station (Genomic Solutions, Ann Arbor) and hybridization was performed at $60^{\circ} \mathrm{C}$ for $50 \mathrm{~min}$. Arrays were then rinsed twice with $2 \mathrm{x}$ SSC, $\mathrm{pH} 4.8,0.1 \%$ SDS, and once with $0.1 \times \mathrm{SSC}, \mathrm{pH} 4.8$. The arrays were exposed to ${ }^{3} \mathrm{H}$-specific phosphorimaging plates (Fuji Medicals) for at least 21 days. Signal intensities for each tRNA species were quantified from eight replicate spots.

\section{Identification of alanine-substituted residues by mass spectrometry}

We performed data analysis of amino acid substitution in the affinity purified, flag-CaMKII protein from HEK293T cells in our mass spectrometry data, which has been reported previously ${ }^{11}$. The MS/MS data of CaMKII protein was generated in the Proteomics and Mass Spectrometry Facility at Donald Danforth Plant Science Center (St. Louis, MO). Protein samples were reacted with iodoacetamide to block all free cysteines before injected into mass spectrometer. In our mass spec data, all detected Cys residues were fully protected and show up in our peptide spectra as carboamidemethyl-Cys (CAM). Tryptic digestion was performed at $37^{\circ} \mathrm{C}$ in $100 \mathrm{ng} / \mu 1$ trypsin, $50 \mathrm{mM}$ ammonium bicarbonate $(\mathrm{pH} 7.8)$ at a ratio of a 1:25 (protease:protein sample). Desulferization of Cys to Ala unlikely occurred in our experiment as TCEP was absent ${ }^{12}$.

To search for alanine-substituted peptides, FASTA sequences of CaMKII were created by substituting other 19 amino acids to alanine residue. Mascot ${ }^{13}$ and MaxQuant ${ }^{14}$ softwares were used to identify peptides containing alanine substitutions. Mascot was searched with a fragment ion mass tolerance of $0.80 \mathrm{Da}$ and a parent ion tolerance of 15 PPM assuming the digestion enzyme trypsin. Carbamidomethylation of cysteine was specified in Mascot as fixed modification. Deamidated of asparagine and glutamine and oxidation of methionine were specified as variable modifications. All spectra containing alanine-mistranslated residues with mass shift were manually inspected, resulting in the identification of a Cys-to-Ala substituted peptide. 


\section{Identification of G4U69 containing tRNA genes in archaea and eukaryotic genomes}

All annotated tRNA genes from archaea (80 species) and eukaryotes (150 species) in the Genomic tRNA database ${ }^{15}$ were searched for G4:U69 containing tRNAs using custom Python scripts. The search was specified for the fourth nucleotide as $G$ from the 5' end $\left(\mathrm{xxxG}_{)}\right)$and the fifth nucleotide as T from the 3' end (Txxx). Some archaea species also have 3'CCA encoded in their tRNA genes; therefore, archaeal searches were also done for 3' end TxxxxCCA. All hits were manually inspected for accuracy. Only tRNAs with tRNAScan score of $>50$ are included in subsequent analysis, as these tRNAs have a high likelihood to form the standard, cloverleaf secondary structure. 


\section{Supplementary Figures}

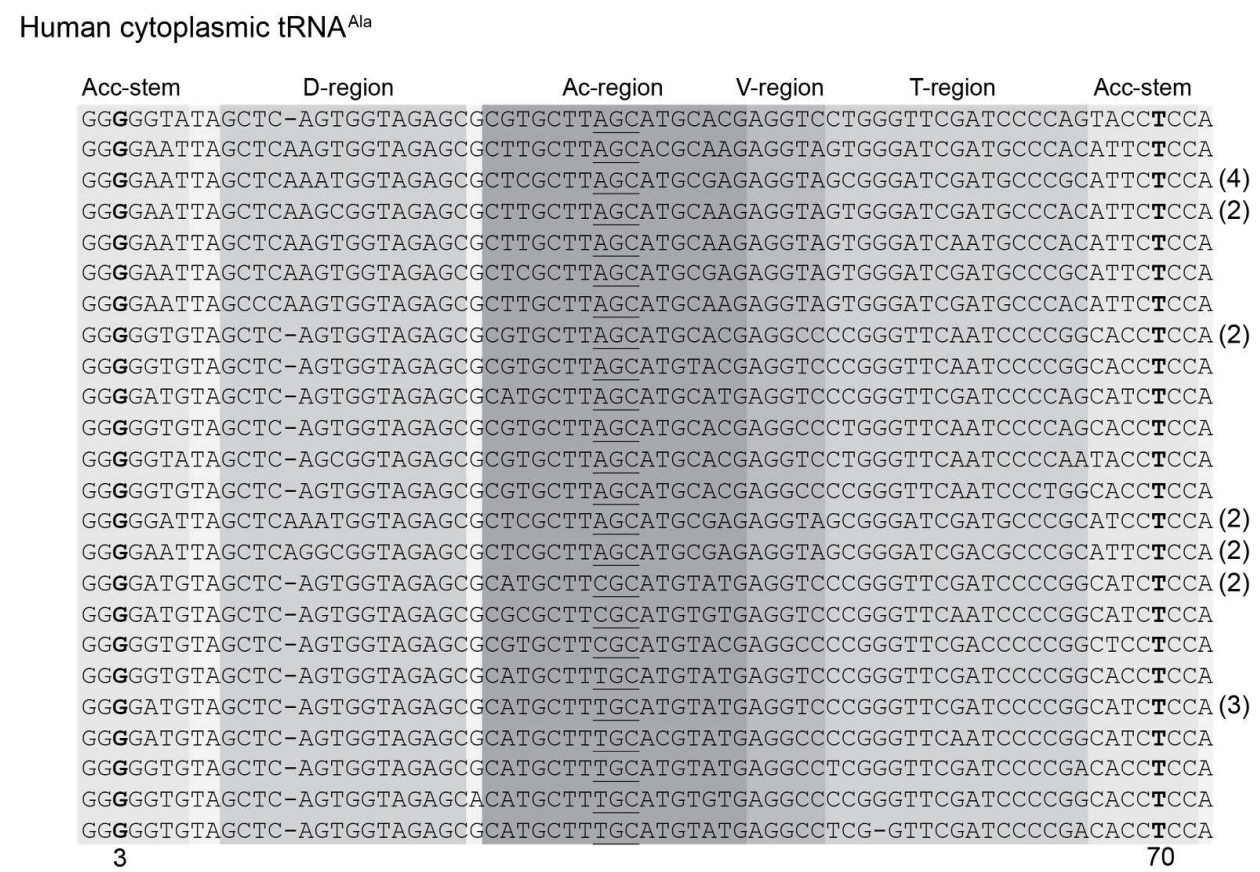

\section{Supplementary Figure 1. Human cytoplasmic tRNA ${ }^{\text {Ala }}$ gene sequences.}

tRNA genes with tRNAScan score of $>50$ that indicates high likelihood to form cloverleaf secondary structure are shown. Different regions of the tRNA are indicated. G3 and T70 (to give the G3:U70 base pair in tRNA ${ }^{\mathrm{Ala}}$ ) are shown in bold and the anticodon nucleotides are underlined.

The number of the tRNA genes in the human genome with the same sequence is shown at the end in brackets. 


\title{
a. Human cytoplasmic tRNAcys
}

\begin{abstract}
Acceptor stem D stem loop Anticodon Variable loop T stem loop Acceptpr stem GGGGGTAGGGCTCAGG-GATAGAGCATTTGACTGCAGATCAAGAGGTCCCCGGTTCGAATCTAGGTGCCCCCT GGGG:ATAGCTCACA-GGTAGAGCATTTGACTGCAGATCAAGAGGTCCCCGGTTCAAATCCGGTTAqTCCCT GGGCGTATAGCTCAGG-GGTAGAGCATTTGACTGCAGATCAAGAGGTCCCCAGTTCAAATCTGGGTGCCCACT GGGGGTATAGCTCACA-GGTAGAGCATTTGACTGCAGATCAAGAGGTCCCCGGTTCAAATCTGGGTGCCCCCT GGGCGTATAGCTCAGG-GGTAGAGCATTTGACTGCAGA TCAAGAGGTCCCCAGTTC.AAATCTGGGTGCCCCCT GGGGGTATAGCTCAGG-GGTAGAGCATTTGACTGCAAATCAAGAGGTCCCTGATTCAAATCCAGGTGCCCCCT GGGGGTATAGTTCAGG-GGTAGAGCATTTGACTGCAGATCAAGAGGTCCCTGGTTCAAATCCAGGTGCCCCCT GGGGATATAGCTCAGG-GGTAGAGCATTTGACTGCAGATCAAGAGGTCCCCGGTTCAAATCCGGGTGCCCCCC GGGGGTATAGCTCAGG GGTAGAGCACTTGACTGCAGATCAAGAAGTCCTTGGTTCAAATCCAGGTGCCCCCT GGGGGTATAGCTCAGG-GGTAGAGCATTTGACTGCAGATCAAGAGGTCTCTGGTTCAAATCCAGGTGCCCCCT GGGGGTATAGCTCAGG-GGTAGAGCATTTGACTGCAGATCAAGAAGTCCCCGGTTCAAATCCGGGTGCCCCCT GGGGGTATAGCTCAGG-GGTAGAGCATTTGACTGCAGATCAAGAGGTCCCCAGTTCAAATCTGGGTGCCCCCT

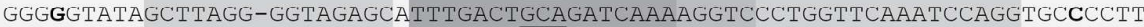
GGGGGTATAGCTTAGC-GGTAGAGCATTTGACTGCAGATCAAGAGGTCCCCGGTTCAAATCCGGGTGCCCCCT GGGGGTATAGCTCAGG-GGTAGAGCATTTGACTGCAGATCAAGAGGTCCCTGGTTCAAATCCAGGTGCCCCCC GGGGGTATAGCTCAGG-GGTAGAGCATTTGACTGCAGATCAAGAGGTCCCTGGTTCAAATCCAGGTGCCCCCT (4) GGGGGTATAGCTCAGG-GGTAGAGCATTTGACTGCAGATCAAGAGGTCCCCGGTTCAAATCCGGGTGCCCCCT GGGGGTATAGCTCAGGTGGTAGAGCATTTGACTGCAGATCAAGAGGTCCCCGGTTCAAATCCGGGTGCCCCCT GGGGGTGTAGCTCAGT-GGTAGAGCATTTGACTGCAGATCAAGAGGTCCCTGGTTCAAATCCAGGTGCCCCCT GGGGGTATAGCTCAGTGGGTAGAGCATTTGACTGCAGATCAAGAGGTCCCCGGTTCAAATCCGGGTGCCCCCT GGGGGTATAGCTCAGT-GGTAGAGCATTTGACTGCAGATCAAGAGGTCCCTGGTTCAAATCCGGGTGCCCCCT GGGGGTATAGCTCAGG-GGTAGAGCACTTGACTGCAGATCAAGAGGTCCCTGGTTCAAATCCAGGTGCCCCCT GGGGGTATAGCTCAGT-GGTAGAGCATTTGACTGCAGATCAAGAGGTCCCCGGTTCAAATCCGGGTGCCCCCT (4) GGGGGCATAGCTCAGTG-GTAGAGCATTTGACTGCAGATCAAGAGGTCCCTGGTTCAAATCCAGGTGCCCCCT
\end{abstract}

4

69

\section{b. Human cytoplasmic tRNA ${ }^{\text {Thr }}$}
Acceptor stem D stem loop
Anticodor
Variable loop
T stem loop
Acceptpr stem GGdG_CGTGGCTTAGTTGGTTAAAGCGCCTGTCTAGTAAACAG-GAGATCCTGGGTTCGAATCCCAGCGETFCCT (3) GGCTCCGTGGCTTAGCTGGTTAAAGCGCCTGTCTAGTAAACAG-GAGATCCTGGGTTCGAATCCCAGCGGGGCCT (2) GGCTCCGTAGCTTAGTTGGTTAAAGCGCCTGTCTAGTAAACAG-GAGATCCTGGGTTCGACTCCCAGCGGGGCCT GGCTTCGTGGCTTAGCTGGTTAAAGCGCCTGTCTAGTAAACAG-GAGATCCTGGGTTCGAATCCCAGCGAGGCCT GGdGCGTGGCTTAGCTGGTTAAAGCGCCTGTCTAGTAAACAG-GAGATCCTGGGTTCGAATCCCAGCGGTFCCT GGCCCTGTGGCTTAGCTGGTCAAAGCGCCTGTCTAGTAAACAG-GAGATCCTGGGTTCGAATCCCAGCGGGGCCT GGCTCTATGGCTTAGTTGGTTAAAGCGCCTGTCTCGTAAACAG-GAGATCCTGGGTTCGACTCCCAGTGGGGCCT GGdGGGTGGCCAAGT--GGTAAGGCGTCGGTCTCGTAAACCG-AAGATCACGGGTTCGAACCCCGTCCETFCCT GGCTCTGTGGCTTAGTTGGCTAAAGCGCCTGTCTCGTAAACAG-GAGATCCTGGGTTCGAATCCCAGCGGGGCCT GGOGFGTGGCCAAGT--GGTAAGGCGTCGGTCTCGTAAACCG-AAGATCGCGGGTTCGAACCCCGTCCAT GCCT GGCCCTGTAGCTCAGC-GGTTGGAGCGCTGGTCTCGTAAACCTAGGGGTCGTGAGTTCAAATCTCACCAGGGCCT GGCTCTATGGCTTAGTTGGTTAAAGCGCCTGTCTTGTAAACAG-GAGATCCTGGGTTCGAATCCCAGTAGAGCCT GGCTCCATAGCTCAGT-GGTTAGAGCACTGGTCTTGTAAACCA-GGGGTCGCGAGTTCGATCCTCGCTGGGGCCT GGCTCCATAGCTCAGG-GGTTAGAGCGCTGGTCTTGTAAACCA-GGGGTCGCGAGTTCAATTCTCGCTGGGGCCT GGCTCCATAGCTCAGG-GGTTAGAGCACTGGTCTTGTAAACCA-GGGGTCGCGAGTTCAAATCTCGCTGGGGCCT GGCCCTATAGCTCAGG-GGTTAGAGCACTGGTCTTGTAAACCA-GGGGTCGCGAGTTCAAATCTCGCTGGGGCCT GGCTCCATAGCTCAGG-GGTTAGAGCACTGGTCTTGTAAACCA-GG-GTCGCGAGTTCAAATCTCGCTGGGGCCT 4

c. Human mitochondrial tRNA Asp

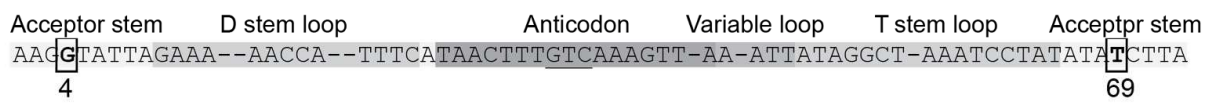

\section{Supplementary Figure 2. Human cytoplasmic tRNA ${ }^{\text {Cys }}$, tRNA $^{\text {Thr }}$ and mitochondrial tRNA ${ }^{\text {Asp }}$}

\section{gene sequences.}

tRNA genes with tRNAScan score of $>50$ that indicates high likelihood to form cloverleaf secondary structure are shown. Different regions of the tRNA are indicated. Position 4 and position 69 are shown in bold and the anticodon nucleotides are underlined. The number of the tRNA genes in the human genome with the same sequence is shown at the end in brackets. G4 and T69 (to give the G4:U69 base pair) are boxed. 
1 exclusive unique peptides, 1 exclusive unique spectra, 251 total spectra, $358 / 478$ amino acids $(75 \%$ coverage)

MAT ITATRFT EEYQLFEELG KGAFSVYRRC VKVLAGQEYA AKIINTKKLS ARDHQKLERE ARICRLLKHP NIVRLHDSIS

EEGHHYLIFD LVTGGELFED IVAREYYSEA DASHCIQQIL EAVLHCHQMG VVHRDLKPEN LLLASKLKGA AVKLADFGLA

IEVEGEQQAW FGFAGTPGYL SPEVLRKDPY GKPVDLWACG VILYILLVGY PPFWDEDQHR LYKQIKAGAY DFPSPEWDTV

TPEAKDLTNK MLTINPSKR TAAEALKHPW ISHRSTVASC MHRQETVDCL KKFNARRKLK GAILTTMLAT RNFSGGKSGG

NKKSDGVKES SESTNTTIED EDTKVRKQEI IKVTEQLIEA ISNGDFESYT KMCDPGMTAF EPEALGNLVE GLDFHRFYFE
NLWSRNSKPV HTTILNPHIH LMGDESACIA YIRITQYLDA GGIPRTAQSE ETRVWHRRDG KWQIVHFHRS GAPSVLPH

b

MATITCTRFTEEYQLFEELGKGAFSVVRRCVKVLAGQEYAAKI INTKKLSARDHQKLEREARICRLLKHPN

IVRLHDS ISEEGHHYLI FDLVTGGELFEDIVAREYYSEADASHCIQQI LEAVLHCHQMGVVHR ( 6 ) DLKPE

NLLLASKLKGAAVKLADFGLAIEVEGEQQAWFGFAGTPGYLSPEVLRKDPYGKPVDLWACGVILY ILLVGY

PPFWDEDQHRLYK (11) QIKAGAYDFPS PEWDTVTPEAKDLINKMLTINPSKRITAAEALKHPWISHRSTV

ASCMHRQETVDCLKKFNAR (2) RKLKGAILTTMLATRNFSGGKSGGNKKSDGVKESSESTNTTIEDEDTKV

RKQEIIKVTEQLIEAISNGDFESYTKMCDPGMTAFEPEALGNLVEGLDFHR (5) FYFENLWSRNSKPVHTT

ILNPHIHLMGDESACIAYIRITQYLDAGGIPR (3) TAQSEETRVWHRRDGKWQIVHFHRSGAPSVLPH

c

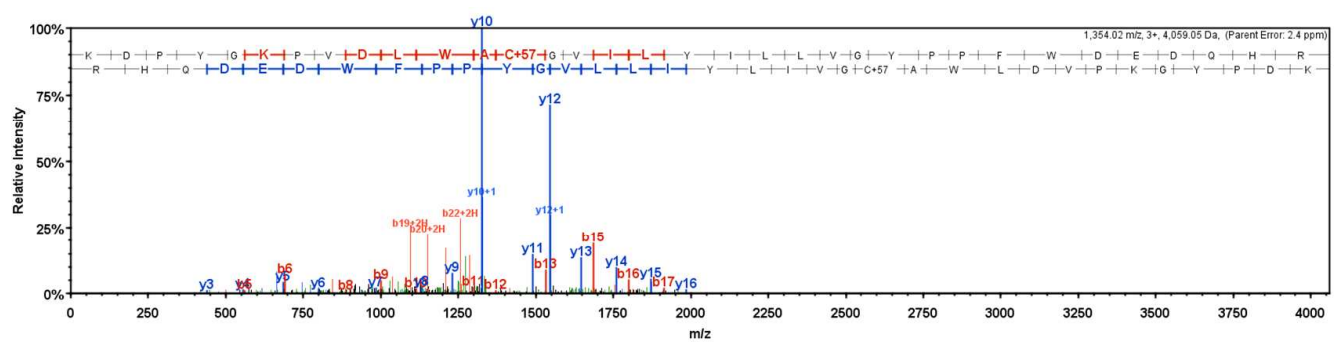

Supplementary Figure 3. Mass spectrometry for Cys-to-Ala search in the CaMKII protein.

(a) The CaMKII protein sequence covered in the mass spec experiment ( $75 \%$ of total) is highlighted in yellow. Modified amino acid side chains detected are shown in green. Six of the ten Cys residues in CaMKII could be directly detected, all in modified form (carbamidomethyl) derived from iodoacetamide reaction prior to injection into mass spectrometer. (b) The precise peptide sequences (underlined) that contain Cys residues and the number of spectra for each peptide are shown in parenthesis following the sequence. Red indicates Cys residues covered by the identified peptides, while blue indicates the uncovered Cys residues. (c) An example of the MS-MS spectrum showing b and y ions of the peptide KDPYGKPVDLWAcGVILYILLVGYPPFWDEDQHR (c = carbamidomethyl, +57 in mass). 
a

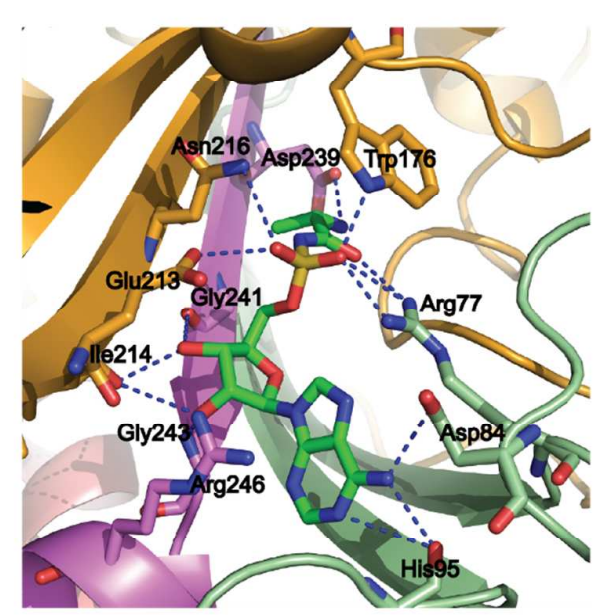

b

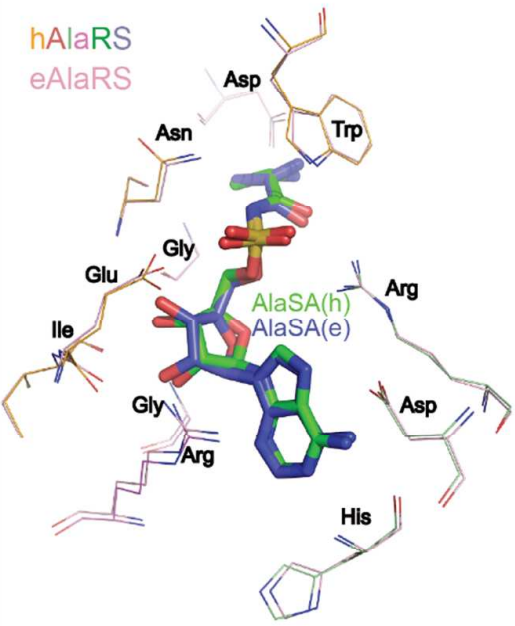

Supplementary Figure 4. The interactions of AlaSA in the active site of human AlaRS.

(a) H-bonding interactions between AlaSA and active site residues of human AlaRS. AlaRS is shown in ribbons (with the same color scheme as in Figure 4a) and AlaSA is shown in sticks (green, carbon; red, oxygen; blue, nitrogen; yellow, phosphorus). (b) The AlaSA interactions are highly conserved between human and E. coli AlaRS. Active site residues of E. coli AlaRS are shown in pink, whereas those of human AlaRS follow the same color scheme as above and as in Figure 4a. 


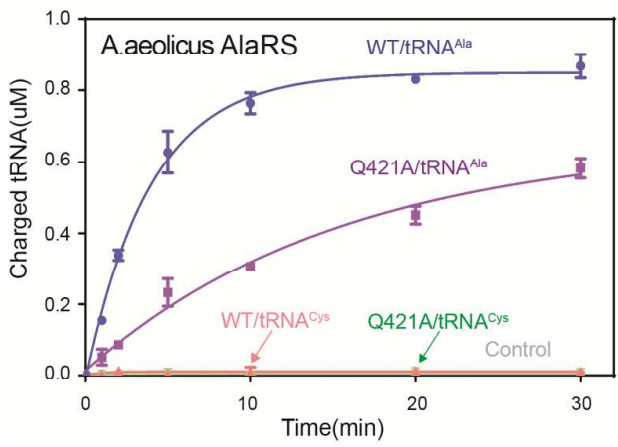

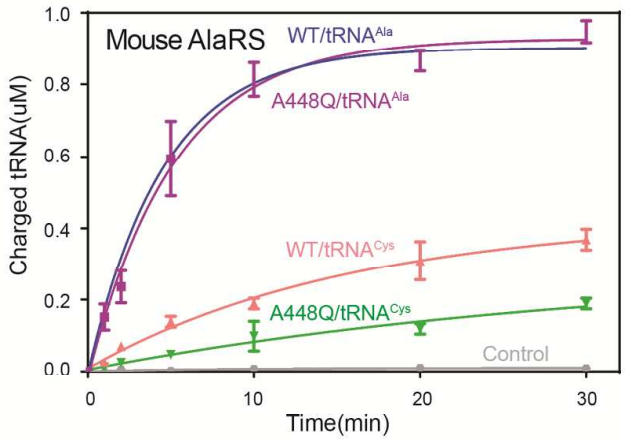

Supplementary Figure 5. In vitro aminoacylation assay with A.aeolicus and mouse AlaRS.

(a) Gln-to-Ala (Q421A) substitution in A.aeolicus AlaRS is unable to mischarge human G4:U69-tRNA ${ }^{\text {Cys }}$. Assays were carried out with $200 \mathrm{nM}$ of WT or Q421A AlaRS, and $2.5 \mu \mathrm{M}$ tRNA $^{\text {Ala }}$ or $25 \mu \mathrm{M}$ G4:U69-tRNA ${ }^{\text {Cys }}$. (b) Ala-to-Gln (A448Q) substitution reduces the mischarging activity of mouse AlaRS towards G4:U69-tRNA ${ }^{\text {Cys }}$. The aminoacylation assays were carried out with $200 \mathrm{nM}$ of WT or A448Q AlaRS, and $2.5 \mu \mathrm{M}$ tRNA $^{\text {Ala }}$ or $10 \mu \mathrm{M}$ G4:U69-tRNA ${ }^{\text {Cys }}$. 


\section{References}

(1) Otwinowski, Z;; Minor, W. Method Enzymol 1997, 276, 307.

(2) Vagin, A.; Teplyakov, A. J Appl Crystallogr 1997, 30, 1022.

(3) Emsley, P.; Cowtan, K. Acta Crystallogr D Biol Crystallogr 2004, 60, 2126.

(4) Murshudov, G. N.; Vagin, A. A.; Dodson, E. J. Acta Crystallogr D 1997, 53, 240.

(5) Adams, P. D.; Afonine, P. V.; Bunkoczi, G.; Chen, V. B.; Davis, I. W.; Echols, N.; Headd, J. J.; Hung, L. W.; Kapral, G. J.; Grosse-Kunstleve, R. W.; McCoy, A. J.; Moriarty, N. W.; Oeffner, R.; Read, R. J.; Richardson, D. C.; Richardson, J. S.; Terwilliger, T. C.; Zwart, P. H. Acta Crystallogr D Biol Crystallogr 2010, 66, 213.

(6) Laskowski, R. A.; Macarthur, M. W.; Moss, D. S.; Thornton, J. M. J Appl Crystallogr 1993, 26, 283.

(7) Beebe, K.; Waas, W.; Druzina, Z.; Guo, M.; Schimmel, P. Anal Biochem 2007, $368,111$.

(8) Xu, X. L.; Shi, Y.; Zhang, H. M.; Swindell, E. C.; Marshall, A. G.; Guo, M.; Kishi, S.; Yang, X. L. Nat Commun 2012, 3, 681.

(9) Netzer, N.; Goodenbour, J. M.; David, A.; Dittmar, K. A.; Jones, R. B.; Schneider, J. R.; Boone, D.; Eves, E. M.; Rosner, M. R.; Gibbs, J. S.; Embry, A.; Dolan, B.; Das, S.; Hickman, H. D.; Berglund, P.; Bennink, J. R.; Yewdell, J. W.; Pan, T. Nature 2009, 462, 522.

(10) Jones, T. E.; Alexander, R. W.; Pan, T. Proc Natl Acad Sci U S A 2011, 108, 6933.

(11) Wang, X.; Pan, T. PLoS Genet 2015, 11, e1005745.

(12)Wang, Z.; Rejtar, T.; Zhou, Z. S.; Karger, B. L. Rapid Commun Mass Spectrom 2010, 24, 267.

(13)Koenig, T.; Menze, B. H.; Kirchner, M.; Monigatti, F.; Parker, K. C.; Patterson, T.; Steen, J. J.; Hamprecht, F. A.; Steen, H. J Proteome Res 2008, 7, 3708.

(14)Cox, J.; Mann, M. Nat Biotechnol 2008, 26, 1367.

(15)Chan, P. P.; Lowe, T. M. Nucleic Acids Res 2016, 44, D184. 